\title{
BMJ Open Synthesising evidence regarding hospital to home transitions supported by volunteers of third sector organisations: a scoping review protocol
}

\author{
Michelle LA Nelson (D) , ${ }^{1,2,3}$ Alana Armas, ${ }^{1}$ Rachel Thombs, ${ }^{1}$ Hardeep Singh, ${ }^{1}$ \\ Joseph Fulton, ${ }^{1}$ Heather V Cunningham, ${ }^{4}$ Sarah Munce, ${ }^{2,5,6}$ Sander Hitzig, ${ }^{6,7}$ \\ Janet Prvu Bettger (iD ${ }^{8}$
}

To cite: Nelson MLA, Armas A, Thombs R, et al. Synthesising evidence regarding hospital to home transitions supported by volunteers of third sector organisations: a scoping review protocol. BMJ Open 2021;11:e050479. doi:10.1136/ bmjopen-2021-050479

- Prepublication history and additional supplemental material for this paper are available online. To view these files, please visit the journal online (http://dx.doi.org/10.1136/ bmjopen-2021-050479).

Received 03 March 2021 Accepted 16 June 2021

A Check for updates

(c) Author(s) (or their employer(s)) 2021. Re-use permitted under CC BY-NC. No commercial re-use. See rights and permissions. Published by BMJ.

For numbered affiliations see end of article.

Correspondence to Dr Michelle LA Nelson; Michelle.Nelson@ sinaihealthsystem.ca

\section{ABSTRACT}

Introduction Given the risks inherent in care transitions, it is imperative that patients discharged from hospital to home receive the integrated care services necessary to ensure a successful transition. Despite efforts by the healthcare sector to develop health system solutions to improve transitions, problems persist. Research on transitional support has predominantly focused on services delivered by healthcare professionals; the evidence for services provided by lay navigators or volunteers in this context has not been synthesised. This scoping review will map the available literature on the engagement of volunteers within third sector organisations supporting adults in the transition from hospital to home.

Methods and analysis Using the well-established scoping review methodology outlined by the Joanna Briggs Institute, a five-stage review is outlined: (1) determining the research question, (2) search strategy, (3) inclusion criteria, (4) data extraction and (5) analysis and presentation of the results. The search strategy will be applied to 10 databases reflecting empirical and grey literature. A two-stage screening process will be used to determine eligibility of articles. To be included in the review, articles must describe a community-based programme delivered by a third sector organisation that engages volunteers in the provisions of services that support adults transitioning from hospital to home. All articles will be independently assessed for eligibility, and data from eligible articles will be extracted and charted using a standardised form. Extracted data will be analysed using narrative and descriptive analyses.

Ethics and dissemination Ethics approval is not required for this scoping review. Members of an international special interest group focused on the voluntary sector will be consulted to provide insight and feedback on study findings, help with dissemination of the results and engage in the development of future research proposals. Dissemination activities will include peer-reviewed publications and academic presentations.

\section{INTRODUCTION}

Transitioning from hospital to home is well recognised to be a time of increased vulnerability. ${ }^{1-9}$ People who may already be
Strengths and limitations of this study

- Application of a rigorous, well-established methodological framework will ensure production of a highquality review.

- A comprehensive search will be conducted on 10 disciplinary and cross-disciplinary databases to ensure that we will maximise our coverage of all possible records that meet review inclusion criteria.

- Inclusion of grey literature further strengthens our review by reducing publication bias and enhancing comprehensiveness of the findings.

- To facilitate a timely review given the high number of citations, the synthesis will be limited to articles published in English which increases the risk of missing relevant programmes reported in languages other than English.

vulnerable, such as the elderly, immigrants and individuals with complex chronic conditions, are even more so during transitions and may require additional support. ${ }^{9-13}$ By definition, a transition of care is 'a set of actions designed to ensure the coordination and continuity of healthcare as patients transfer between different locations or different levels of care within the same location. ${ }^{14}$ Irrespective of the care trajectory a person follows, they will undergo at least one transition during their time as an inpatient, either from one care setting or level of care to another, or through discharge back home. ${ }^{14-16}$ Transitions are inherently complex, requiring well-coordinated efforts by many individuals across multiple settings. ${ }^{14} 17$ Ideally, transitions involve comprehensive care plans that include community follow-up and timely information exchange between hospital and community practitioners about a patient's treatment goals, preferences and their health and clinical status. ${ }^{17-20}$ However, in reality, 
there is growing evidence of failures in provider communication and fragmented care during care transitions. ${ }^{19-21}$ Fragmentation can lead to adverse events, including medication errors, readmissions, decreased patient satisfaction, further morbidity and even mortality. ${ }^{22-27}$ These issues can be exacerbated in communities where resources and services are lacking, transportation limitations exist, providers are limited or wait times are increased. ${ }^{626-31}$ Given the risks associated with transitions, ${ }^{1-9}$ it is imperative that those discharged home from hospital receive the care and assistance necessary to ensure that their care transition is safe and successful. Despite efforts of the healthcare sector to develop health system solutions to improve transitions, problems persist, such as poor communication, organisation of services, provision of medication and poor quality of discharge instructions from one setting to another. ${ }^{32} 33$

Efforts to address issues related to transitions of care are occurring at all levels in the health and social care sectors. Increasingly complex patient populations, ${ }^{34}$ new reimbursement models, ${ }^{35}$ shortened lengths of hospital stay $^{36}$ and pressures to reduce costs while improving care quality and patient experience have driven policy makers to seek innovative solutions. The Health Systems Learning Group, comprised of 43 healthcare organisations from across the USA, noted that 'as hospitals and health systems struggle under the weight of uncompensated care, emergency department (ED) overuse, and readmissions-the greater portion directly attributable to spiraling chronic disease-the case for transformative community partnerships becomes increasingly clear' (p65) ${ }^{37}$ Spurred by health reforms, health system leaders at the macro level have turned to partnerships with third sector organisations (TSOs) to improve patient care. ${ }^{38}$ 'Third sector organisations' refers to those that are neither public nor private sector. The term includes voluntary and community organisations (registered charities and other organisations such as associations, self-help groups and community groups) and social enterprises. ${ }^{39} 40$ TSOs are situated within local communities and are focused on meeting the needs of community members through non-profit generating activities. Since they are embedded in the communities they serve, TSOs have a deep understanding of community members' needs and are in a trusted position to help. ${ }^{41}$ Partnerships with and between TSOs are a recognised way to expand the breadth and quality of health and social services. ${ }^{42-44}$ It has been suggested that partnering with TSOs is an effective way for a welfare state to augment their activities because the knowledge, skill and innovation inherent to TSOs can be drawn on when designing and delivering communitybased programmes and services. ${ }^{40}{ }^{45}$ For example, the 'Memphis Model' is a partnership between more than 600 congregations and Methodist Le Bonheur Healthcare to support congregants post-discharge through the collaborative efforts of hospital-employed navigators and trusted volunteers from each parish. ${ }^{46}$ This model is seen to create synergy between different types of organisations focused on health and well-being, ultimately being of value to patients, congregations and community more broadly. ${ }^{47}$ However, the role and contributions of a key community partner-TSOs-in improving patient experience and system efficiency are still often unaccounted for, or under-recognised. ${ }^{47}$

While the partnerships between health system leaders and TSOs represent engagement at the macro and meso levels, it is the volunteers contributing towards successful transitions who are working at the micro level. Volunteers are defined as individuals who undertake activities or provide services to others as part of a defined role without remuneration for their time, effort or talent. ${ }^{48}$ They are also considered a health human resource by the WHO which includes any individual working in the public or private sectors on a part-time or full-time basis and who are paid or provide services on a volunteer basis. ${ }^{49}$ The term volunteer is not used to refer to support services provided by unpaid family or friend caregivers. One such defined role for volunteers working in health services is the lay navigator role. Lay navigators are individuals without a health profession background who are recruited from the community and receive specialised training to assist patients in navigating complex health and social care systems and minimise barriers to accessing care. ${ }^{50}$ Lay navigators contribute to successful transitions post-hospital discharge by helping people maintain health service engagement through assistance with scheduled appointments and referrals, providing accompaniment to appointments, communicating with relevant agencies and organisations and assisting with paperwork and forms. ${ }^{51-53}$ In addition, lay navigators also sign post and help adults with chronic illness living in the community build connections with their community with the intent to improve quality of life, and develop independence and engagement. ${ }^{54}$

Recognising the potential value of voluntary sector services in supporting patient care, organisations such as The Kings Fund, ${ }^{55}$ the Institute for HealthCare Improvement ${ }^{56}$ and The Beryl Institute ${ }^{57}$ have advocated for more purposeful engagement of TSOs in the organisation and delivery of healthcare. Research on the engagement of volunteers in defined roles and partnerships with TSOs as forms of integrated care during care transitions is emerging, but the extent and nature of this evidence is unclear. ${ }^{58-60}$ To address this knowledge gap, this scoping review will map the available literature focused on the contributions of third sector personnel and services to support transitions from hospital to home. In doing so, study results will inform future service development and identify areas of future research.

\section{METHODS AND ANALYSIS}

Published literature on community-based volunteer supported transition programmes is likely to appear in a variety of sources and vary in methodological approaches and formats. Using the methods outlined in the Joanna 
Briggs Institute manual for knowledge synthesis ${ }^{61}$ and the Preferred Reporting Items for Systematic Reviews and Meta-Analysis extension for Scoping Reviews (PRISMA-ScR) guidelines ${ }^{62}$ (see online supplemental appendix 1) will allow us to make use of literature across study designs and in both peer-reviewed and grey literature. ${ }^{63}$ The study will be completed over five stages: (1) determining the research question, (2) search strategy, (3) inclusion criteria, (4) data extraction and (5) analysis and presentation of the results. Though the study will not entail a quality assessment, scoping reviews are considered a rigorous and systematic approach to knowledge synthesis. Study activities will occur between 2021 and 2022. Ethics Review Board approval is not required for the conduct of this study.

\section{Determining the research question}

Members of this research team had previously convened experts to identify high priority research questions specific to rehabilitation for stroke patients with multiple concurrent health and social issues. ${ }^{64}$ During this consensus meeting, several questions arose about the role and potential of 'non health sector' organisations ${ }^{64}$ which informed the development of the research question for this scoping review. Furthermore, members of an international special interest group focused on transitional care interventions highlighted additional knowledge needs regarding third sector interventions to support transitions from hospital to home. Based on feedback from these groups, the study question was not limited to the stroke population, since programmes developed for other patient populations may be transferable. This review will answer the following research question: How, where, and for which populations have third sector organisations engaged volunteers in programmes supporting adults in the transition from hospital to home?

Study objectives include:

1. To determine in which geographical (ie, countries) and health service areas (i.e. geriatrics, mental health, chronic conditions, rehabilitation) volunteers of TSOs have been engaged to support adults in the transition home after hospital discharge.

2. To document programme characteristics of transitional models (i.e., funding, staffing ratios, training, service parameters) delivered by volunteers of TSOs.

3. To identify characteristics of clients of (i.e., age, condition, gender, race/ethnicity) participating in community-based volunteer supported transition programmes.

4. To identify relevant knowledge gaps that can support the development of a research programme focused on transitions and community reintegration with volunteers as a key service provider.

\section{Search strategy}

To identify relevant peer-reviewed studies, the research team, along with an experienced information scientist (HVC), developed a comprehensive search strategy. The initial search strategy was generated for Ovid MEDLINE and peer reviewed by Information Science Librarians, after which the Information Scientist adopted the search to the MeSH terms and concepts for the remaining databases. Due to the nature of the research question, the team recognised that potentially relevant literature would be identified in a wide variety of databases. After careful consideration, nine additional databases were selected for the review: EMBASE, PsycInfo, Joanna Briggs (JBI), Social Work Abstracts, Sociological Abstracts, CINAHL, Cochrane Reviews, Ageline and Scopus. The preliminary search of all databases identified 19720 records. Reference lists and bibliographies of the identified articles will also be searched for citations not identified by the database search. A grey literature search will be conducted to identify any non-indexed literature of relevance. All literature searches will be conducted by the information scientist (HVC) on the study team. Finally, other global experts in transitions will be consulted in a 'desk drawer' search strategy to ensure that all relevant citations are obtained. The studies included in the review will be amalgamated and stored using reference management software package, EndNote, to ensure there are no duplicates in the database. Please see online supplemental appendix 2 for the complete Medline search strategy.

\section{Inclusion criteria}

The inclusion criteria for this scoping review support the collection and extraction of data relevant to the research question and objectives. Following the JBI construct of population, concept and context, the inclusion criteria are as follows:

\section{Population}

Any service designed to support adults (age 18+) transitioning from hospital to home will be eligible. As the focus of this review is on programme characteristics of transitional support interventions, the review will include all volunteer-supported hospital to home transition programmes available to any adult population, to ensure the search strategy retrieves the widest pool of relevant literature. Programmes and services offered to children and adolescents will not be included as these programmes are expected to be designed and implemented to address different needs and may require the inclusion of parents and guardians. As such, these differences constitute a separate review of the literature that goes beyond the scope of the current review.

\section{Concept}

Volunteer-supported transitions from hospital to home. Transitional care interventions with volunteer workforce will be included as the transition from hospital to home will is the focus of this review. As various terms have been used to describe the process of hospital discharge and return to home and community settings, search terms will include post discharge, transition*, after-care, posthospital, community reintegration. The WHO included 
volunteers in definitions of Health Human Resources. ${ }^{65}$ For this review, volunteers are defined as individuals who undertake activities or provide services to others as part of a formal role within a TSO, without remuneration for their time or talent. ${ }^{48}$

\section{Context}

Any programme provided within a 'third sector' organisation will be eligible for inclusion. The third sector describes organisations that are non-government, nonprofit, charitable, faith based or are a social enterprise.

\section{Date range}

2000-present. We have limited the search to literature published since 2000 , in part to ensure we have sufficient relevant citations, balanced with synthesising programme characteristics that are still relevant in current approaches to the organisation and delivery of health services.

\section{Types of evidence to be included}

Published and unpublished literature reporting any quantitative, qualitative, mixed or multi methods research, including both comparative (eg, randomised, controlled, cohort, quasi-experimental) and non-comparative (eg, survey, narrative, audit) methods, educational materials and reports. We will include any study design. Reference lists of relevant knowledge syntheses will be searched for relevant articles. Grey literature may include unpublished research, programme summaries, evaluation reports, theses, organisational reports and conference proceedings. Literature will be limited to only those published in English.

Once all identified records have been extracted from all databases and duplicates have been removed, the data will be uploaded into knowledge synthesis management platform, Covidence, for screening and extraction. The inclusion and exclusion criteria will be tested on a randomly selected set of citations. Once the final inclusion and exclusion criteria have been determined, the team will pull another random sample of identified articles to screen and test inter-rater reliability, using the Kappa coefficient. We will continue to test inter-rater reliability until a Kappa of 0.85 (ie, excellent agreement) ${ }^{66}$ is reached. Title and abstract screening will be conducted in duplicate on all identified articles. Based on the inclusion and exclusion criteria, the reviewers will categorise the articles as 'Yes', 'No' or 'Maybe'; all 'Yes' and 'Maybe' articles will be included for full-text screening. Any discrepancies will be reviewed and resolved by the senior research team members and experts in this field. Interrater reliability will be continuously tested throughout the title and abstract screen to ensure a high rating is maintained. This will also give the research team multiple opportunities to discuss and resolve discrepancies. For full-text screening, the reviewers will categorise the articles as either 'Yes' or 'No', and any uncertainties will be discussed by the team with discrepancies adjudicated by a senior member of the research team.

\begin{tabular}{|c|c|}
\hline Category & Data to be extracted \\
\hline $\begin{array}{l}\text { Article } \\
\text { information }\end{array}$ & $\begin{array}{l}\text { Author, journal/publication source, year of } \\
\text { publication, publication type (ie, academic/ } \\
\text { scientific paper, grey literature, editorial, } \\
\text { press release, organisation report, etc), } \\
\text { study/programme location }\end{array}$ \\
\hline $\begin{array}{l}\text { Study design (if } \\
\text { applicable) }\end{array}$ & $\begin{array}{l}\text { Research question, study objectives/aims, } \\
\text { study design, methods }\end{array}$ \\
\hline $\begin{array}{l}\text { Organisation } \\
\text { profile }\end{array}$ & $\begin{array}{l}\text { Organisation size (eg, local group, national } \\
\text { or international), geographic location, } \\
\text { type of organisation, affiliations with other } \\
\text { organisations (eg, community organisation } \\
\text { working with a local hospital) }\end{array}$ \\
\hline $\begin{array}{l}\text { Programme } \\
\text { characteristics }\end{array}$ & $\begin{array}{l}\text { Programme aims/objectives, health } \\
\text { service context (ie, rehabilitation, primary } \\
\text { care, public health), transitional services } \\
\text { provided, delivery mechanisms, eligibility } \\
\text { criteria, service administration (funding, } \\
\text { staffing mix, volunteer requirements, } \\
\text { training, etc) }\end{array}$ \\
\hline $\begin{array}{l}\text { Client and } \\
\text { volunteer } \\
\text { characteristics }\end{array}$ & $\begin{array}{l}\text { Client characteristics (ie, age, condition, } \\
\text { gender, race/ethnicity), volunteer } \\
\text { characteristics (ie, age, condition, gender, } \\
\text { race/ethnicity), type of volunteer (student, } \\
\text { peer, health professional, retirees, etc) }\end{array}$ \\
\hline $\begin{array}{l}\text { Evaluation and } \\
\text { outcomes (if } \\
\text { applicable) }\end{array}$ & $\begin{array}{l}\text { Programme evaluation procedures, } \\
\text { programme outcomes, client outcomes }\end{array}$ \\
\hline
\end{tabular}

\section{Data extraction}

A copy of each article/document will be obtained, reviewed and charted by two research team members. Data extraction will be completed by specified research team members for all articles (ie, two researchers per article, with adjudication by a third researcher) using an extraction form that will be pilot tested before use. During pilot testing, extraction criteria may be modified for full extraction of the included articles. Any discrepancies that occur during extraction will be reviewed and resolved by a senior member of the research team. Table 1 outlines the preliminary data extraction plan for the study.

\section{Critical appraisal of available literature}

As the primary aim of this scoping review is to provide an overview of the existing literature, we will not undertake a formal quality assessment of each study. We will, however, assign a 'level of evidence' rating to each citation using JBI's well-established categories. ${ }^{61}$ This level of analysis will allow for an evaluation of the types of research that has been undertaken in terms of established evidence hierarchies, and to comment on the existing evidence base as a whole.

\section{Analysis and presentation of results}

A scoping review is designed to provide an overview of the extent and nature of a body of literature. To do this, we will employ three reporting and presentation strategies: (a) a 
modified PRISMA-SR, ${ }^{62}$ (b) a basic numerical account of the amount, type and distribution of the studies included in the review (addressing objective 1) and (c) a thematic analysis and visual representations of included literature reporting key data extraction categories (ie, geographic distribution of programmes, programme characteristics, volunteer management processes, client characteristics) which address objectives 2 and 3 .

The specific reporting products will be organised and their structure determined by the results and needs of our identified knowledge users. Identified knowledge users for our research findings include TSO programme developers, researchers and health system leaders focused on hospital discharge and transitions from hospital to home. The anticipated breadth of the literature spanning conditions, setting, age and location makes it challenging to adopt an existing conceptual framework to comprehensively map the literature. As a result, we anticipate that we will have to develop a framework to best summarise and present the results of this review.

\section{Knowledge translation}

Throughout study conduct, we will consult members of an existing international special interest group focused on volunteers and the third sector in the design and delivery of integrated care; a network led by members of the research team. This international research, policy and practice-oriented group will provide insight and feedback on study findings, help with dissemination of the results, and engage in the development of future research proposals. Traditional end of grant dissemination activities, including peer-reviewed publications and academic presentations at local, national and international conferences are planned.

\section{PATIENT AND PUBLIC INVOLVEMENT}

Patients or the public were not involved in the design, conduct, reporting or dissemination of our research.

\section{ETHICS AND DISSEMINATION}

Throughout data extraction and analysis of the included studies, we will consult with members of an existing international special interest group focused on the voluntary sector in integrated care. This group will provide insight and feedback on study findings, help with dissemination of the results, and engage in the development of future research proposals. Traditional end of grant dissemination activities, including peer reviewed publications and academic presentations at local, national and international conferences are planned. As this is a scoping review protocol, ethics approval is not applicable.

\section{Author affiliations}

${ }^{1}$ Bridgepoint Collaboratory for Research and Innovation, Lunenfeld-Tanenbaum Research Institute; Sinai Health, Toronto, Ontario, Canada
${ }^{2}$ Institute of Health Policy, Management and Evaluation, Dalla Lana School of Public Health, University of Toronto, Toronto, Ontario, Canada

${ }^{3}$ Knowledge to Action, March of Dimes Canada, Toronto, Ontario, Canada

${ }^{4}$ Gerstein Science Information Centre, University of Toronto, Toronto, Ontario, Canada

${ }^{5}$ LIFEspan Service, KITE, Toronto Rehabilitation Institute; University Health Network, Toronto, Ontario, Canada

${ }^{6}$ Faculty of Medicine, Department of Occupational Science and Occupational Therapy, University of Toronto, Toronto, Ontario, Canada

${ }^{7}$ St. John's Rehab Research Program, Sunnybrook Research Institute, Sunnybrook Health Sciences Centre, Toronto, Ontario, Canada

${ }^{8}$ Global Health Institute, Duke University, Durham, North Carolina, USA

\section{Twitter Michelle LA Nelson @mlanelson}

Contributors MLAN conceptualised and designed the study, wrote and critically revised the protocol and manuscript. AA and RT wrote and critically revised the protocol and manuscript, and collaborated with the information scientist on the search strategies. HS, SH, JPB and SM critically revised the protocol and manuscript. JF collaborated with the information scientist on the grey literature search strategy and revised the protocol and manuscript. As the information scientist, HVC devised the peer-reviewed and grey literature search strategies in collaboration with the team.

Funding The authors have not declared a specific grant for this research from any funding agency in the public, commercial or not-for-profit sectors.

Competing interests None declared.

Patient consent for publication Not required.

Provenance and peer review Not commissioned; externally peer reviewed.

Supplemental material This content has been supplied by the author(s). It has not been vetted by BMJ Publishing Group Limited (BMJ) and may not have been peer-reviewed. Any opinions or recommendations discussed are solely those of the author(s) and are not endorsed by BMJ. BMJ disclaims all liability and responsibility arising from any reliance placed on the content. Where the content includes any translated material, BMJ does not warrant the accuracy and reliability of the translations (including but not limited to local regulations, clinical guidelines, terminology, drug names and drug dosages), and is not responsible for any error and/or omissions arising from translation and adaptation or otherwise.

Open access This is an open access article distributed in accordance with the Creative Commons Attribution Non Commercial (CC BY-NC 4.0) license, which permits others to distribute, remix, adapt, build upon this work non-commercially, and license their derivative works on different terms, provided the original work is properly cited, appropriate credit is given, any changes made indicated, and the use is non-commercial. See: http://creativecommons.org/licenses/by-nc/4.0/.

\section{ORCID IDs}

Michelle LA Nelson http://orcid.org/0000-0003-2002-0298 Janet Prvu Bettger http://orcid.org/0000-0001-9708-8413

\section{REFERENCES}

1 Neiterman E, Wodchis WP, Bourgeault IL. Experiences of older adults in transition from hospital to community. Can J Aging 2015;34:90-9.

2 Naylor MD. Transitional care for older adults: a cost-effective model. LDI Issue Brief 2004;9:1-4.

3 Krumholz HM. Post-hospital syndrome--an acquired, transient condition of generalized risk. N Engl J Med 2013;368:100-2.

4 Foust JB, Naylor MD, Bixby MB, et al. Medication problems occurring at hospital discharge among older adults with heart failure. Res Gerontol Nurs 2012;5:25-33.

5 Andreasen J, Lund $\mathrm{H}$, Aadahl M, et al. The experience of daily life of acutely admitted frail elderly patients one week after discharge from the hospital. Int J Qual Stud Health Well-being 2015;10:27370.

6 Jencks SF, Williams MV, Coleman EA. Rehospitalizations among patients in the Medicare fee-for-service program. N Engl J Med 2009;360:1418-28.

7 Mesquita ET, Cruz LN, Mariano BM, et al. Post-Hospital syndrome: a new challenge in cardiovascular practice. Arq Bras Cardiol 2015;105:540-4.

8 van Seben R, Reichardt LA, Essink DR, et al. "I Feel Worn Out, as if I Neglected Myself": Older Patients' Perspectives on Post- 
hospital Symptoms After Acute Hospitalization. Gerontologist 2019:59:315-26.

9 Hestevik CH, Molin M, Debesay J, et al. Older persons' experiences of adapting to daily life at home after hospital discharge: a qualitative metasummary. BMC Health Serv Res 2019;19:224.

10 Parekh AK, Goodman RA, Gordon C, et al. Managing multiple chronic conditions: a strategic framework for improving health outcomes and quality of life. Public Health Rep 2011;126:460-71.

11 Baxter R, Shannon R, Murray J, et al. Delivering exceptionally safe transitions of care to older people: a qualitative study of multidisciplinary staff perspectives. BMC Health Serv Res 2020;20:780.

12 Rustad EC, Furnes B, Cronfalk BS, et al. Older patients' experiences during care transition. Patient Prefer Adherence 2016;10:769-79.

13 Tomlinson J, Cheong V-L, Fylan B, et al. Successful care transitions for older people: a systematic review and meta-analysis of the effects of interventions that support medication continuity. Age Ageing 2020;49:558-69.

14 Coleman EA, Boult C, American Geriatrics Society Health Care Systems Committee. Improving the quality of transitional care for persons with complex care needs. J Am Geriatr Soc 2003;51:556-7.

15 Naylor M, Keating SA. Transitional care. Am J Nurs 2008;108:58-63.

16 Coleman EA, Smith JD, Frank JC, et al. Preparing patients and caregivers to participate in care delivered across settings: the care transitions intervention. J Am Geriatr Soc 2004;52:1817-25.

17 Waring J, Marshall F, Bishop S. Health Services and Delivery Research. An ethnographic study of knowledge sharing across the boundaries between care processes, services and organisations: the contributions to 'safe' hospital discharge. Southampton, UK: NIHR Journals Library, 2014.

18 Cohen MD, Hilligoss PB. The published literature on handoffs in hospitals: deficiencies identified in an extensive review. Qual Saf Health Care 2010;19:493-7.

19 Abrashkin KA, Cho HJ, Torgalkar S, et al. Improving transitions of care from hospital to home: what works? Mt Sinai J Med 2012;79:535-44.

20 Clarke JL, Bourn S, Skoufalos A, et al. An innovative approach to health care delivery for patients with chronic conditions. Popul Health Manag 2017;20:23-30.

21 Abraham J, Kannampallil T, Patel B, et al. Ensuring patient safety in care transitions: an empirical evaluation of a handoff intervention tool. AMIA Annu Symp Proc 2012;2012:17-26.

22 Laugaland K, Aase K, Barach P. Addressing risk factors for transitional care of the elderly - literature review, 2011. Available: https://www.researchgate.net/publication/267834203_Addressing Risk_Factors_for_Transitional_Care_of_the_Elderly_-_Literature _ review

23 Riesenberg LA, Leitzsch J, Cunningham JM. Nursing handoffs: a systematic review of the literature. Am J Nurs 2010;110:24-34. quiz 35-6.

24 Kripalani S, LeFevre F, Phillips CO, et al. Deficits in communication and information transfer between hospital-based and primary care physicians: implications for patient safety and continuity of care. JAMA 2007;297:831-41.

25 Coleman EA. Falling through the cracks: challenges and opportunities for improving transitional care for persons with continuous complex care needs. J Am Geriatr Soc 2003;51:549-55.

26 Danzl MM, Harrison A, Hunter EG, et al. "A Lot of Things Passed Me by": Rural Stroke Survivors' and Caregivers' Experience of Receiving Education From Health Care Providers. J Rural Health 2016:32:13-24

27 Lustig D, Weems GH, Strauser D. Rehabilitation service patterns: a rural/urban comparison of success factors. J Rehabil 2004;70:13-19.

28 Institute of Medicine (US) Committee on Quality of Health Care in America. Crossing the quality chasm: a new health system for the 21st century. Washington, DC: National Academies Press (US), 2001

29 Deber RB. Health care reform: lessons from Canada. Am J Public Health 2003;93:20-4.

30 Soril LJJ, Adams T, Phipps-Taylor M, et al. Is Canadian healthcare affordable? A comparative analysis of the Canadian healthcare system from 2004 to 2014. Healthc Policy 2017;13:43-58.

31 MacNeil M, Koch M, Kuspinar A, et al. Enabling health technology innovation in Canada: barriers and facilitators in policy and regulatory processes. Health Policy 2019;123:203-14.

32 Rochester-Eyeguokan CD, Pincus KJ, Patel RS, et al. The current landscape of transitions of care practice models: a scoping review. Pharmacotherapy 2016;36:117-33.

33 Markiewicz O, Lavelle M, Lorencatto F, et al. Threats to safe transitions from hospital to home: a consensus study in North West London primary care. Br J Gen Pract 2020;70:bjgp19X707105.
34 Kingston A, Comas-Herrera A, Jagger C, et al. Forecasting the care needs of the older population in England over the next 20 years: estimates from the population ageing and care simulation (PACSim) modelling study. Lancet Public Health 2018;3:e447-55.

35 Chapin RK, Chandran D, Sergeant JF, et al. Hospital to community transitions for adults: discharge planners and community service providers' perspectives. Soc Work Health Care 2014;53:311-29.

36 Bueno H, Ross JS, Wang Y, et al. Trends in length of stay and shortterm outcomes among Medicare patients hospitalized for heart failure, 1993-2006. JAMA 2010;303:2141-7.

37 Stakeholder Health. Health systems learning group monograph. Washington, DC, 2013. https://stakeholderhealth.org/pdf/

38 Garrett D, Hwang A. CBOs and state Medicaid programs: a key partnership for patient-centered care. Generations 2018;42:19-23.

39 Corry O. Defining and theorizing the third sector. In: Taylor R, ed. Third sector research. New York, NY: Springer New York, 2010: 11-20.

40 Defourny J. Third sector to social enterprise. In: Borzaga CJD, ed. The emergence of social enterprise. London and New York: Routledge, 2001.

41 Dickinson H, Allen K, Alcock P. The role of the third sector in delivering social care, 2012. Available: http://eprints.Ise.ac.uk/ 43538/1/The\%20role\%20of\%20the\%20third\%20sector\%20in\% 20delivering\%20social\%20care.pdf

42 Brinkerhoff D, Brinkerhoff J. Public-private partnerships: perspectives on purposes, publicness, and good governance. . Public Administration and Development, 2011: 31. 2-14.

43 Ejaz I, Shaikh BT, Rizvi N. NGOs and government partnership for health systems strengthening: a qualitative study presenting viewpoints of government, NGOs and donors in Pakistan. BMC Health Serv Res 2011:11:122.

44 Hushie M. Public-non-governmental organisation partnerships for health: an exploratory study with case studies from recent Ghanaian experience. BMC Public Health 2016;16:963.

45 Bode I, Brandsen T. State-third sector partnerships: a short overview of key issues in the debate. Public Management Review 2014;16:1055-66.

46 Cutts TBB. Church-health system partnership facilitates transitions from hospital to home for urban, low-income African Americans, reducing mortality, utilization, and costs. service delivery innovation profile. AHRQ Innovations, 2012. Available: http://www.innovations. ahrq.gov/content.aspx?id=3354

47 Cutts T. The Memphis model: ARHAP theory comes to ground in the congregational health network. Pietermaritzburg: Cluster Publications, 2010: 193-206.

48 Cnaan RA, Handy F, Wadsworth M. Defining who is a volunteer: conceptual and empirical considerations. Nonprofit Volunt Sect $Q$ 1996;25:364-83.

49 WHO. Toolkit on monitoring health systems strengthening: human resources for health, 2009. Available: https://www.who.int/healthinfo/ statistics/toolkit_hss/EN_PDF_Toolkit_HSS_HumanResources_oct08. pdf [Accessed 24 Apr 2021].

50 Toal-Sullivan D, Lemonde M, Gauthier AP, et al. Adopting a lay navigator training programme in primary care. Health Educ $J$ 2021;80:210-24.

51 Carter N, Valaitis R, Feather J, et al. An environmental scan of health and social system navigation services in an urban Canadian community. SAGE Open Nurs 2017;3:237796081668956.

52 Corrigan P, Sheehan L, Morris S, et al. The impact of a peer navigator program in addressing the health needs of Latinos with serious mental illness. Psychiatr Serv 2018;69:456-61.

53 Maxwell AE, Jo AM, Crespi CM, et al. Peer navigation improves diagnostic follow-up after breast cancer screening among Korean American women: results of a randomized trial. Cancer Causes Control 2010;21:1931-40.

54 Pesut B, Duggleby W, Warner G, et al. Volunteer navigation partnerships: Piloting a compassionate community approach to early palliative care. BMC Palliat Care 2017;17:2.

55 Imison CBR. NHS and social care workforce: meeting our needs now and in the future. London: The Kings Fund, 2013. https:// www.kingsfund.org.uk/sites/default/files/field/field_publication_file/ perspectives-nhs-social-care-workforce-jul13.pdf

56 American Hospital Association. Engaging health care volunteers to Pursue the triple Aim. Chicago: American Hospital Association, 2017. https://www.aha.org/content/17/17engagingvolunteerstrip leaim.pdf

57 Garrison MWJ. The role of the volunteer in improving patient experience Nashville. The Beryl Institute, 2016. https://www. theberylinstitute.org/store/ViewProduct.aspx?id=6373089

58 Manderson B, McMurray J, Piraino E, et al. Navigation roles support chronically ill older adults through healthcare transitions: 
a systematic review of the literature. Health Soc Care Community 2012;20:113-27.

59 Lorhan S, Cleghorn L, Fitch M, et al. Moving the agenda forward for cancer patient navigation: understanding volunteer and peer navigation approaches. J Cancer Educ 2013;28:84-91.

60 Egan M, Anderson S, McTaggart J. Community navigation for stroke survivors and their care partners: description and evaluation. Top Stroke Rehabil 2010;17:183-90.

61 Aromataris EMZ. JBI manual for evidence synthesis, 2020

62 Tricco AC, Lillie E, Zarin W, et al. PRISMA extension for scoping reviews (PRISMA-ScR): checklist and explanation. Ann Intern Med 2018;169:467-73.
63 Peters MDJ GC, Mclnerney P, Munn Z, et al. Chapter 11: Scoping Reviews. In: Aromataris EMZ, ed. JBI manual for evidence synthesis. $\mathrm{JBI}, 2020$.

64 Nelson ML, McKellar KA, Munce S, et al. Addressing the evidence gap in stroke rehabilitation for complex patients: a preliminary research agenda. Arch Phys Med Rehabil 2018;99:1232-41.

65 World Health Organization. Human resources for health, 2009. Available: https://www.who.int/healthinfo/statistics/toolkit_hss/EN PDF_Toolkit_HSS_HumanResources_oct08.pdf [Accessed 24 Apr 2021].

66 McHugh ML. Interrater reliability: the kappa statistic. Biochem Med 2012;22:276-82 